\title{
Evidências de validade dos escores estratégicos na tarefa de fluência verbal infantil
}

\author{
Priscila do Nascimento Marques ${ }^{1}$, Jane Correa, Rosinda Martins Oliveira \\ Universidade Federal do Rio de Janeiro, Rio de Janeiro-RJ, Brasil \\ Helenice Charchat-Fichman \\ Pontifícia Universidade Católica, Rio de Janeiro-RJ, Brasil
}

\section{RESUMO}

Os escores estratégicos na tarefa de fluência verbal fonológica (FVF) são utilizados na avaliação das funções executivas e linguísticas de crianças, e dois métodos para pontuação são empregados: valor bruto (VB) e taxas (TX). Existem poucas evidências de sua validade. Este trabalho objetivou descrever as evidências de validade de VB e TX, considerando o modelo de busca estratégica na FVF. Administrou-se a FVF em 106 crianças saudáveis de nove a 12 anos. Nas análises de regressão stepwise (VB), os três escores estratégicos foram preditores significativos do número total de emissões. No TX, apenas a taxa do tamanho médio de agrupamentos foi preditora. Nas análises de componente principal, VB gerou dois fatores (organização e flexibilidade); enquanto TX, apenas um fator bipolar (organização). Concluiu-se que VB mostrou mais evidências de validade haja vista o modelo multifatorial de busca estratégica, que pressupõe habilidades executivas de organização e flexibilidade.

Palavras-chave: crianças; psicometria; função executiva; neuropsicologia.

\section{ABSTRACT - Validity evidence for strategic scores on Verbal Fluency tasks in childhood}

Strategic scores on phonological verbal fluency (PVF) tasks are used in the evaluation of executive and language skills of children, and two methods of scoring are employed: raw scores (RS) and rates (R). There is little evidence of validity for each. This paper aimed to describe the validity evidences of GV and R considering the strategic search model in PVF. The PVF tasks were administered in 106 healthy children of nine to 12 years old. In stepwise regression analysis (GV), the three strategic scores were significant predictors of total emissions. In R, only the mean cluster size was predictive. In principal component analysis, GV generated two factors (organization and flexibility); while R, only generated one bipolar factor (organization). In conclusion, GV showed more evidence of validity considering the multifactorial model of strategic search, which assumes executive organization skills and flexibility. Keywords: children; psychometrics; executive function; neuropsychology.

\section{RESUMEN - Evidencia de validez de puntaje estratégico en la tarea de Fluencia Verbal infantil}

Los puntajes estratégicos en la tarea de fluidez verbal fonológica (FVF), se utilizan en la evaluación de habilidades ejecutivas y lingüísticas de los niños, y se evalúan teniendo en cuenta el valor bruto (VB) y tasa (TX). Hay pocas evidencias de validez para cada uno. Este documento tiene como objetivo describir dicha validez, teniendo en cuenta el modelo de búsqueda estratégica en FVF. Se les administró la FVF a 106 niños saludables de nueve a 12 años. En el análisis de regresión por pasos (VB), los tres puntajes estratégicos son predictores significativos de las emisiones totales. En TX, solo el tamaño medio de las agrupaciones fue predictivo. En el análisis de componentes principales VB generó dos factores (organización y flexibilidad), mientras que TX, solo uno bipolar (organización). Concluimos que VB mostró más evidencia de validez, teniendo en cuenta el modelo multifactorial de investigación estratégica, que asume las habilidades ejecutivas de organización y flexibilidad.

Palabras clave: niños; psicometría; función ejecutiva; neuropsicología.

Tarefas de fluência verbal semântica (FVS) e fonológica (FVF) são comumente usadas em Neuropsicologia, tanto no contexto clínico quanto no de pesquisa (Lezak, Howieson, Bigler, \& Tranel, 2012). Na FVS, o sujeito produz, em um intervalo limitado de tempo, o máximo possível de palavras que pertençam a dada categoria, enquanto na FVF emite palavras que comecem com determinada letra (Strauss, Sherman, \& Spreen, 2006).
A mensuração do desempenho nessas tarefas conta com dois principais sistemas de avaliação: o número de palavras geradas corretamente em cada categoria semântica ou fonológica (Lezak et al., 2012; Strauss et al., 2006); e os escores estratégicos (Raskin, Sliwinski, \& Borod, 1992; Troyer, Moscovitch, \& Winocur, 1997), que descrevem as estratégias de busca no acesso lexical (Chertkow \& Bub, 1990). 
Três escores estratégicos têm sido mais utilizados com adultos (Abwender, Swan, Bowerman, \& Connolly, 2001) e crianças (Nieto, Galtier, Barroso, \& Espinosa, 2008; Tallberg, Carlsson, \& Lieberman, 2011; Takács, Kóbor, Tárnok, \& Csépe, 2014):

1. Número de agrupamentos de palavras produzidas sucessivamente que compartilham características semânticas (pertencentes a uma subcategoria semântica) ou fonológicas (iniciadas ou terminadas com a mesma sílaba);

2. Tamanho médio de agrupamentos, que contabiliza o número médio de palavras emitidas, em sucessão, em uma mesma subcategoria;

3. Número de transições, que contabiliza o número de vezes em que há mudança para uma nova subcategoria de busca (Nieto et al., 2008).

Há evidências de que a FVS e a FVF apresentam natureza multifatorial, sendo o desempenho nas tarefas determinado por processos executivos (organização e flexibilidade, mensurado pelos escores número de agrupamentos e número de transições, respectivamente) e de vocabulário (mensurado pelo escore tamanho médio de agrupamento) (Ruff, Light, \& Parker, 1997; Shao, Janse, Visser, \& Meyer, 2014). Esses resultados são oriundos de estudos com adultos saudáveis (Troyer, 2000) e portadores de lesões nos lobos frontal e temporal (Troyer, Moscovitch, Winocur, Alexander, \& Stuss, 1998) do cérebro.

Mais recentemente, investigações com crianças também apontaram para evidências de validade clínica e da contribuição do modelo multifatorial, contabilizado pelos escores estratégicos. Reportou-se correlação significativa entre o escore número de transições e medidas clássicas de flexibilidade cognitiva (Snyder \& Munakata, 2010). Em grupos clínicos com comprometimento das funções executivas na infância (fenilcetonúria e transtorno do déficit de atenção e hiperatividade), a redução da flexibilidade na busca de palavras, evidenciada pelo escore de transição, mostrou-se determinante para o número total de palavras produzidas (Banerjee, Grange, Steiner, \& White, 2011; Gonçalves, 2015). Já o tamanho médio de agrupamento se correlacionou às medidas de vocabulário e ao funcionamento dos lobos temporais (Kavé, Kigel, \& Kochva, 2008).

No contexto clínico, os escores estratégicos parecem oferecer informações além daquelas fornecidas pelo número de palavras produzidas nas tarefas de fluência verbal (Koren, Kofman, \& Berger, 2005). Crianças com transtorno do espectro autista diferenciaram-se de um grupo controle em termos de escores de número de transições (flexibilidade), mas não quanto ao número de palavras. Enquanto crianças controle foram capazes de produzir agrupamentos e flexibilizar entre novas categorias de busca, crianças do espectro autista geravam agrupamentos; no entanto eram menos habilidosas ao flexibilizar entre novas categorias, obtendo menor número de transições (Begeer et al., 2014).
Apesar das evidências de validade e da investigação crescente dos escores estratégicos e de sua contribuição para a avaliação cognitiva, não há um método unificado para o cálculo nos estudos com crianças, tendo sido mais utilizados:

1. método de pontuação das taxas, que divide cada escore estratégico (número de agrupamentos, número de transições e tamanho médio de agrupamento) pelo número total de palavras produzidas na tarefa, gerando, respectivamente: taxa de agrupamentos, taxa de transições e taxa do tamanho médio de agrupamento (Sauzéon, Lestage, Raboutet, N'Kaoua, \& Claverie, 2004);

2. método de pontuação pelo valor bruto dos escores estratégicos, que não realiza transformação nos escores estratégicos (Troyer et al., 1997).

Ambas as formas de pontuação contabilizam os mesmos escores estratégicos (número de agrupamentos, número de transições e tamanho médio de agrupamentos), porém pontuam esses escores de maneiras diferentes.

A pontuação das taxas dos escores estratégicos (Sauzéon et al., 2004) é pioneira no estudo desses escores em crianças. A rationale ao cálculo de tais taxas subjacente é que dividir os escores estratégicos pelo número total de palavras na tarefa possibilita uma medida mais pura de desenvolvimento executivo. Isto é, o aumento no número de palavras produzidas na FVS e FVF ao longo das idades pode estar correlacionado ao aumento do vocabulário com o decorrer da escolarização, e não ao desenvolvimento de estratégias de organização e a funções executivas, como se supõe (Riva, Nichelli, \& Devoti, 2000).

A pontuação do valor bruto dos escores estratégicos adota cálculo semelhante ao que é utilizado com adultos (Raskin et al., 1992; Troyer et al., 1997), e a maior parte dos estudos com crianças posteriores a Sauzéon et al. (2004) usa tal método. O argumento para o emprego desse tipo de cálculo é o de que a divisão pelo número total de palavras pode criar uma medida artificial, que não traduz o desempenho real do examinando (Koren et al., 2005; Troyer, 2000). Apesar de esse método de pontuação ser o mais empregado, ele deixa em aberto a questão de que o aumento no desempenho observado nos escores estratégicos se deva somente à expansão do vocabulário, e não ao incremento do uso de estratégias.

A investigação de cada um desses métodos de pontuação, enquanto medidas apropriadas para uso com crianças, é necessária, visto que os referidos métodos têm produzido resultados divergentes que conduzem a explicações distintas sobre o desenvolvimento cognitivo infantil. A literatura aponta algumas semelhanças entre os dois métodos de cálculo, em certos aspectos, como, por exemplo: tanto para o valor bruto como para a taxa dos escores estratégicos se reporta aumento linear dos escores de transição ao longo da idade para a FVF e também para a FVS.

Entretanto a maioria dos estudos conduz a outras conclusões que diferem em função do método de cálculo 
(Nieto et al., 2008; Sauzéon et al., 2004). Enquanto no método de taxa o tamanho médio de agrupamentos decresce ao longo da infância na FVF (Sauzéon et al., 2004), no método do valor bruto esse escore aumenta de modo significativo. No primeiro caso, o resultado tem sido interpretado como o fato de que a capacidade de busca em uma mesma subcategoria fonológica não acompanha o crescimento do vocabulário. No segundo caso, o resultado indicaria o contrário (Tallberg et al., 2011).

Outra divergência encontrada é a de que para o método das taxas o número de transições se correlaciona de modo significativo com o número total de palavras apenas na FVS. Isso tem sido interpretado como um papel particularmente importante da flexibilidade das estratégias de busca, quando esta é feita com base em uma categoria semântica (Sauzéon et al., 2004). Já para o método valor bruto, o número de transições contribui de modo significativo para a produção de palavras tanto na FVF como na FVS (Kavé et al., 2008) e sugere que a flexibilidade também determina a busca de palavras por categoria fonológica. As divergências entre as interpretações decorrentes do uso de métodos de pontuação variados para escores estratégicos podem limitar sua validade enquanto medida de funções executivas e habilidades linguísticas (Koren et al., 2005), uma vez que cada método de pontuação explica o desenvolvimento cognitivo infantil de forma diferente.

No Brasil, o interesse pelos escores estratégicos na fluência verbal tem se ampliado nos últimos anos, mas aspectos referentes ao método de pontuação têm sido pouco considerados, e estudos sistemáticos sobre suas qualidades psicométricas ainda são escassos (Abwender et al., 2001), assim como ocorre em diversas medidas em psicologia (Pawlowski, Trentini, \& Bandeira, 2007).

O presente artigo teve como objetivo descrever evidências de validade para cada método de escore estratégico ao interpretar o desempenho de crianças na tarefa de fluência verbal. Foi utilizado como referência o modelo multifatorial de busca estratégica que deu origem a esses escores (Chertkow \& Bub, 1990). O referido modelo define o desempenho na tarefa com base em três processos cognitivos básicos: organização, flexibilidade e extensão do vocabulário (Ruff et al. 1997; Shao et al., 2014). Para tanto, o procedimento adotado analisou evidências de validade fundamentadas na estrutura interna do instrumento (APA, AERA, \& NCME, 1999; Thompson \& Daniel, 1996).

Considerando que a FVF tem se mostrado mais diretamente relacionada às funções executivas em adultos (Andrewe, 2001) e crianças (Kavé et al., 2008; Sauzéon et al., 2004) do que a FVS, o presente artigo investigou os escores estratégicos apenas na FVF. Isso pode permitir melhor comparação entre os dois métodos de cálculo de escores estratégicos, escores estes que parecem depender mais do desenvolvimento das funções executivas que de outras funções cognitivas.

\section{Método}

\section{Participantes}

As crianças incluídas neste estudo fizeram parte do projeto de pesquisa Estudo das Propriedades Psicométricas de Quatro Paradigmas Clássicos em Avaliação Neuropsicológica (Charchat-Fichman, Oliveira, \& Morais, 2011; Oliveira, Mograbi, Gabrig, \& Charchat-Fichman, 2016), aprovado pela Comissão de Ética do Departamento de Psicologia da Pontifícia Universidade Católica do Rio de Janeiro (PUC-RIO). A amostra total foi composta de 396 crianças de sete a 14 anos, sem diagnóstico de doenças neuropsiquiátricas, transtornos de aprendizagem ou histórico de reprovações. Foram excluídos 35 protocolos de crianças que não preencheram os critérios de inclusão. Os participantes frequentavam escolas privadas do Estado do Rio de Janeiro e eram pertencentes às classes socioeconômicas C, D e E (famílias com renda mensal entre uma e cinco vezes o salário mínimo nacional). Essas informações foram coletadas em questionário preenchido pelos pais.

Para melhor análise das propriedades psicométricas dos métodos de cálculo de escores estratégicos, a escolha da faixa etária e escolaridade das crianças considerou o controle da variabilidade no desenvolvimento executivo e no emprego da rota fonológica, que interferem no desempenho na FVF (Luria, 1973; Mousinho \& Correa, 2009). Para isso, foram selecionados aleatoriamente protocolos de crianças de sete a 12 anos de idade $(n=106)$ : 15 protocolos das crianças de sete anos; 37 das de dez anos; 27 das de 11 anos; e 27 das de 12 anos, com escolaridade do $4^{\circ}$ ano até o $7^{\circ}$ ano do ensino fundamental.

\section{Procedimentos}

Cada criança foi avaliada individualmente no horário escolar. O protocolo do estudo original (CharchatFichman et al., 2011; Oliveira et al., 2016) incluiu duas sessões de testagem. Na primeira, eram aplicados Aprendizagem Auditivo-verbal de Rey e Figura Complexa de Rey. A segunda sessão obedecia à seguinte ordem fixa para todos os sujeitos: Tarefa de fluência verbal fonológica (letras F, A, M), Tarefa de fluência verbal semântica (categorias animais, frutas e roupas) e paradigma de Stroop versão Victoria (Charchat-Fichman et al., 2011; Oliveira et al., 2016). Todos os participantes tiveram o Termo de Consentimento Livre e Esclarecido (TCLE) assinado por um dos responsáveis antes de serem iniciadas as testagens.

\section{Tarefa de fluência verbal fonológica}

Foram utilizadas as letras F, A e M em função da transparência ortográfica das palavras que iniciam com a letra $M$ (Martins, Vieira, Loureiro, \& Santos, 2007) e pela maior ocorrência de palavras começadas pela letra $M$ no léxico da língua portuguesa, em comparação à letra $\mathrm{S}$, mais comum em estudos na língua inglesa (Viaro \& Guimarães-Filho, 2007).

$\mathrm{Na}$ administração da FVF, os examinandos foram instruídos a gerar o maior número possível de palavras 
iniciadas com as letras citadas, excluindo nomes próprios e a mesma palavra com terminações diferentes. Foi utilizado um intervalo de 60 segundos para cada letra. A ordem de apresentação das letras foi fixa para todos os sujeitos: F, A, M. As palavras foram gravadas e transcritas na ordem em que foram emitidas pelos examinados.

\section{Pontuação}

Cada protocolo foi corrigido por dois pontuadores independentes, e, em seguida, a concordância entre esses pontuadores foi verificada. Os protocolos que não mostraram concordância foram conferidos por um terceiro pontuador, com o objetivo de minimizar erros de contagem (Abwender et al., 2001). O número de palavras geradas corretamente na FVF foi computado com base em Charchat-Fichman et al. (2011) e Oliveira et al. (2016). Os escores estratégicos foram calculados pelo método valor bruto (Troyer et al., 1997) e taxas dos escores estratégicos (Sauzéon et al., 2004). Para o método valor bruto, calcularam-se os escores número de transições, número de agrupamentos e tamanho médio de agrupamentos conforme Nieto et al. (2008). Foram calculados apenas agrupamentos fonológicos, em função da baixa ocorrência de agrupamentos semânticos na FVF (Tallberg et al., 2011). Para o método das taxas, seguiram-se os critérios estabelecidos por Sauzéon et al. (2004).

A concordância entre pontuadores para o computo do número de palavras corretas emitidas por meio da correlação de Pearson para as letras foi $\mathrm{F}(\mathrm{r}=0,96)$, A $(r=0,97)$ e $\mathrm{M}(r=0,95)$. Para o método de valor bruto dos escores estratégicos (Troyer et al., 1997), a concordância obtida (média F, A e M) foi de: número de agrupamentos $(r=0,95)$; tamanho médio de agrupamentos $(r=0,94)$; e número de transições $(r=0,98)$. Para o método das taxas, a concordância reportada foi equivalente à obtida por Sauzéon et al. (2004).

\section{Análises de dados}

Para análise do desempenho das crianças na FVF, foram utilizadas análises de variância considerando o número de palavras geradas corretamente na FVF, bem como os escores estratégicos pelo método valor bruto (Troyer et al., 1997) e taxas (Sauzéon et al., 2004), em função da idade.

As evidências de validade para cada método de pontuação estratégico (método valor bruto e método das taxas) foram examinadas com base na compatibilidade de cada método com o modelo multifatorial de busca estratégica (em que o desempenho na tarefa é explicado por diferentes processos cognitivos, como organização, flexibilidade e vocabulário), em termos de sua estrutura interna.

Para isso, investigou-se para ambos os métodos o quanto cada escore estratégico contribui para explicar o número de palavras geradas corretamente na FVF, por meio de correlações de Pearson e análises de regressão múltipla pelo método stepwise. Para averiguar a estrutura fatorial, foi realizada análise de componente principal (PCA) para o método valor bruto e para o método taxas.

\section{Resultados}

\section{Desempenho das crianças no número de palavras geradas corretamente e escores estratégicos na FVF}

Número de palavras geradas corretamente na FVF. A média do número de palavras geradas corretamente nas letras $\mathrm{F}, \mathrm{A}$ e $\mathrm{M}$ foi equivalente a 7,90 (desvio padrão $=1,66)$ (Tabela 1). Não houve efeito significativo em razão da letra $\left(F_{(2,204)}=1,96 ; p=0,14\right)$, da idade $\left(F_{(3,102)}=1,53, p=0,33\right)$ nem da interação entre letra e idade $\left(F_{(6,204)}=0,77 ; p=0,59\right)$.

Escores estratégicos. No método valor bruto (Troyer et al., 1997), não foi observado efeito da idade nos escores estratégicos (número de agrupamentos $\left[F_{(3,102)}=0,38 ; p=0,77\right] ;$ tamanho médio de agrupamentos $\left[F_{(3,102)}=0,73 ; \quad p=0,54\right] ; \quad$ número de transições $\left.\left[F_{(3,102)}=1,51 ; p=0,22\right]\right)$. As análises foram conduzidas com as médias dos escores estratégicos obtidas entre as três letras, F, A e M.

Igualmente, não se verificou efeito da idade nas taxas dos escores estratégicos (taxa de agrupamentos $\left[F_{(3,102)}=0,26 ; p=0,85\right]$; taxa do tamanho médio de agrupamentos $\left[F_{(3,102)}=1,29 ; p=0,29\right]$; taxa de transições $\left.\left[F_{(3,102)}=1,35 ; p=0,26\right]\right)$. As médias e os desvios padrão dos escores estratégicos nos métodos valor bruto e taxa encontram-se na Tabela 1.

$\mathrm{Na}$ correlação com a produção total de palavras, todos os escores estratégicos mostraram correlação significativa com o número total de palavras produzidas, tanto no método do valor bruto quanto no de taxas. No método valor bruto, as correlações entre o número total de palavras e cada um dos escores estratégicos foram, respectivamente: número de agrupamentos, $r=0,53(p<0,01)$; número de transições, $r=0,82(p<0,01)$; e tamanho médio de agrupamentos, $r=-0,20(p<0,01)$. No método das taxas dos escores, as correlações foram: taxa de agrupamentos, $r=-0,20(p<0,01)$; taxa de transições, $r=0,39(p<0,01)$; e taxa do tamanho médio de agrupamentos, $r=-0,47(p<0,01)$.

Tabela 1

Médias e desvios padrão dos escores estratégicos na fluência verbal fonológica

\begin{tabular}{|c|c|c|c|c|}
\hline \multicolumn{5}{|c|}{ Escores estratégicos (média FAM) } \\
\hline & \multicolumn{2}{|c|}{$\begin{array}{l}\text { Método } \\
\text { valor bruto }\end{array}$} & \multicolumn{2}{|c|}{$\begin{array}{l}\text { Métodos } \\
\text { das taxas }\end{array}$} \\
\hline & Média & $D P$ & Média & $D P$ \\
\hline Agrupamentos & 1,69 & 0,58 & 0,21 & 0,07 \\
\hline $\begin{array}{l}\text { Tamanho } \\
\text { médio de } \\
\text { agrupamentos }\end{array}$ & 0,60 & 0,57 & 0,09 & 0,10 \\
\hline Transições & 4,77 & 1,54 & 0,59 & 0,13 \\
\hline
\end{tabular}

Nota: FAM=letras F, A e M; DP=desvio padrão. 
Há, no entanto, diferenças quanto à contribuição de cada escore estratégico para o número total de palavras, em cada método. Enquanto no método das taxas a correlação de maior magnitude é entre o total de palavras e a taxa do tamanho médio de agrupamentos $(r=-0,47$, $p<0,01$ ), no método valor bruto o escore que apresenta correlação de maior magnitude com o total de palavras é o número de transições $(r=0,82, p<0,01)$.

\section{Evidências de validade baseadas na estrutura interna para os métodos de pontuação dos escores estratégicos}

Análises de regressão stepwise. Para verificar a contribuição dos escores estratégicos no desempenho total conforme o método do valor bruto e o método das taxas, foram realizadas duas análises de regressão múltipla (método stepwise) para cada método de escore estratégico, tendo como variável dependente o número total de palavras. Embora a idade e a escolaridade não mostrem contribuição significativa para a produção total de palavras, esses fatores foram introduzidos nas etapas iniciais das análises, como forma de controle da variabilidade devida a eles. $\mathrm{Na}$ etapa posterior, foram introduzidos como fatores os três escores estratégicos: número de agrupamentos, número de transições e tamanho dos agrupamentos.
Para o método valor bruto, a entrada das variáveis idade na etapa 1 e escolaridade na etapa 2 não contribuiu de modo significativo (respectivamente, $F_{(1,104)}=3,16 ; p=0,08$; e $\left.F_{(2,103)}=0,09 ; p=0,76\right)$ e explicou de 1 a $3 \%$ a variabilidade na variável dependente. Já a adição do número de transições, na etapa 3, explicou $64 \%$ da variabilidade do número de palavras produzidas e resultou em mudança significativa no modelo $\left(F_{(3,102)}=199,54 ; p<0,01\right)$. Na etapa 4 , o número de agrupamentos introduziu acréscimo de $13 \%$ na explicação da variabilidade $\left(F_{(4,101)}=102,75 ; p<0,01\right)$. Por fim, na etapa 5 , a introdução do tamanho dos agrupamentos provocou acréscimo de $60 \%$ na explicação da variabilidade do número total de palavras, o que produziu mudança significativa $\left(F_{(5,100)}=125,55 ; p<0,01\right)$. Esse modelo predisse $86 \%$ do total da variabilidade do número de palavras geradas corretamente na FVF. A Tabela 2 mostra o resumo da análise de regressão.

Para o método taxas, a entrada da variável idade na etapa 1 e escolaridade na etapa 2 não contribuiu de modo significativo para a explicação da variabilidade no total de palavras (respectivamente, $F_{(1,104)}=3,16 ; p=0,08$; e $\left.F_{(2,103)}=0,09 ; p=0,76\right)$, sendo capaz de explicar de $1 \mathrm{a}$ $3 \%$ a variabilidade no número de palavras corretas produzidas. A adição da taxa do tamanho médio dos agrupamentos na etapa 3 explicou $21 \%$ da variabilidade do número de palavras produzidas, com mudança significativa $\left(F_{(1,102)}=27,57 ; p<0,01\right)$ trazida ao modelo (Tabela 3).

Tabela 2

Resumo da análise de regressão: papel do valor bruto dos escores estratégicos na fluência verbal fonológica

\begin{tabular}{|c|c|c|c|c|c|}
\hline Variável & $B$ & SEB & $\beta$ & $R^{2}$ & $\Delta R^{2}$ \\
\hline \multicolumn{6}{|l|}{ Modelo 1} \\
\hline Idade & 0,12 & 0,07 & 0,17 & 0,03 & 0,030 \\
\hline \multicolumn{6}{|l|}{ Modelo 2} \\
\hline Idade & 0,16 & 0,14 & 0,23 & \multirow{2}{*}{0,03} & \multirow{2}{*}{0,001} \\
\hline Escolaridade & $-0,05$ & 0,15 & $-0,06$ & & \\
\hline \multicolumn{6}{|l|}{ Modelo 3} \\
\hline Idade & $-0,03$ & 0,09 & $-0,04$ & \multirow{3}{*}{0,67} & \multirow{3}{*}{0,640} \\
\hline Escolaridade & 0,07 & 0,09 & 0,09 & & \\
\hline Número de transições & 0,82 & 0,06 & $0,82^{* *}$ & & \\
\hline \multicolumn{6}{|l|}{ Modelo 4} \\
\hline Idade & $-0,04$ & 0,07 & $-0,06$ & \multirow{4}{*}{0,80} & \multirow{4}{*}{0,130} \\
\hline Escolaridade & 0,06 & 0,07 & 0,08 & & \\
\hline Número de transições & 0,74 & 0,05 & $0,74^{* *}$ & & \\
\hline Número de agrupamentos & 0,38 & 0,05 & $0,37^{* *}$ & & \\
\hline \multicolumn{6}{|l|}{ Modelo 5} \\
\hline Idade & $-0,04$ & 0,06 & $-0,04$ & \multirow{5}{*}{0,86} & \multirow{5}{*}{0,600} \\
\hline Escolaridade & 0,03 & 0,06 & 0,04 & & \\
\hline Número de transições & 0,96 & 0,05 & $0,96^{* *}$ & & \\
\hline Número de agrupamentos & 0,29 & 0,04 & $0,28^{* *}$ & & \\
\hline Tamanho de agrupamentos & 0,21 & 0,03 & $0,33^{* *}$ & & \\
\hline
\end{tabular}

Nota: $N=106 ;{ }^{* *} p<0,01 ; B=$ coeficiente de regressão não padronizado; $S E B=$ erro padronizado; $\beta=$ coeficiente de regressão padronizado; $R^{2}=$ coeficiente de determinação com variância explicada; $\Delta R^{2}=$ diferença na proporção da variância explicada. 
Análises de Componente Principal (PCA). Para verificar a natureza dos escores estratégicos em cada método de pontuação de escores estratégicos (valor bruto e taxa) em termos de sua estrutura fatorial interna, foram conduzidas análises exploratórias de Componente Principal (Principal Component Analysis).

No método valor bruto, com base na Análise de Componente Principal (PCA), foram extraídos dois componentes principais, com eigenvalues maiores do que 1, que juntos explicaram $89,52 \%$ da variância total. O primeiro componente (que carrega a variável número de transições) explicou 53,36\% da variância e apresentou eigenvalue de 1,60; e o segundo componente (o qual carrega a variável número de agrupamentos) explicou 36,16\% da variância, com eigenvalue de 1,09. A Tabela 4 mostra a carga de cada escore estratégico pelo método valor bruto em relação a esses dois componentes principais.

Para o método taxas, a PCA permitiu a extração de um único componente apresentando eigenvalue superior a 1. Tal componente (com eigenvalue equivalente a 2,20) explicou $73,35 \%$ da variância total. A Tabela 4 exibe a carga de cada escore estratégico pelo método das taxas em relação ao componente principal. A distribuição das três taxas em um único fator de natureza bipolar revelou que os escores estratégicos, pelo método das taxas, podem ser atribuídos a um único construto, a organização. A Tabela 4 contém a carga de cada escore estratégico pelo método valor bruto no tocante a esses dois componentes principais. Abaixo, há a carga de cada taxa de escore estratégico pelo método das taxas, referente a um único componente principal.

\section{Discussão}

O presente artigo teve como objetivo estudar as evidências de validade baseadas na estrutura interna de dois métodos de escores estratégicos na FVF utilizados com crianças: o método do valor bruto e o método das taxas.

Para o estudo de validade, foi utilizado como referência o modelo que considera a natureza multifatorial das tarefas de fluência verbal, que inclui três processos cognitivos básicos: organização em agrupamentos, extensão de agrupamentos e flexibilidade. Para isso, em cada método de pontuação, foi investigada a importância das categorias dos escores correspondentes a esses processos na explicação da variabilidade no número de palavras corretas evocadas na FVF. Além disso, examinou-se a estrutura fatorial de cada método de pontuação.

Assim como descrito na literatura (Charchat-Fichman et al., 2011; Riva et al., 2000), para a faixa etária estudada, não houve efeito significativo do tipo de letra, de idade e de escolaridade no número total de palavras produzidas. Quanto aos escores estratégicos, as médias e os desvios padrão obtidos para as diferentes idades são consistentes com a literatura, tanto para o método valor bruto (Nieto et al., 2008; Kavé et al., 2008) como para as taxas (Sauzéon et al., 2004).

Entretanto a diferença entre os métodos, em termos da magnitude das correlações dos escores estratégicos com a produção total de palavras, sugere que os diferentes tipos de cálculo provocam mudanças qualitativas nesses escores e conduzem a formas distintas de explicar a contribuição de

Tabela 4

Análise de componente principal (PCA) para o método valor bruto e o método das taxas dos escores estratégicos

\begin{tabular}{lcc}
\hline Método valor bruto & \multicolumn{2}{c}{ Componentes } \\
\cline { 2 - 3 } (Troyer et al., 1997) & \multicolumn{2}{c}{2} \\
\hline Tamanho médio & $-0,88$ & 0,31 \\
de agrupamento & 0,91 & 0,19 \\
Número de transições & 0,11 & 0,98 \\
Número de agrupamentos & \multicolumn{2}{c}{ Componente } \\
\cline { 2 - 3 } Método das taxas & \multicolumn{2}{c}{1} \\
(Sauzéon et al., 2004) & \multicolumn{2}{c}{0,91} \\
\hline Taxa do tamanho & \multicolumn{2}{c}{$-0,94$} \\
médio de agrupamento & \multicolumn{2}{c}{0,71} \\
Taxa de transições & \multicolumn{2}{c}{0} \\
Taxa de agrupamentos & \multicolumn{2}{c}{0}
\end{tabular}

Tabela 3

Resumo da análise de regressão: papel das taxas dos escores estratégicos na fluência verbal fonológica

\begin{tabular}{lccccc}
\hline Variável & $B$ & SEB & $\beta$ & $R^{2}$ & $\Delta R^{2}$ \\
\hline $\begin{array}{l}\text { Modelo 1 } \\
\quad \text { Idade }\end{array}$ & 0,12 & 0,07 & 0,17 & 0,03 & 0,030 \\
$\quad$ Modelo 2 & & & & & \\
$\quad$ Idade & 0,16 & 0,14 & 0,23 & 0,03 & 0,001 \\
$\quad$ Escolaridade & $-0,05$ & 0,15 & $-0,06$ & & \\
$\quad$ Modelo 3 & & & & & \\
$\quad$ Idade & 0,08 & 0,13 & 0,11 & & 0,210 \\
$\quad$ Escolaridade & 0,01 & 0,14 & 0,02 & 0,24 & \\
$\quad$ Taxa do tamanho médio dos agrupamentos & $-0,32$ & 0,06 & $-0,46^{* *}$ & &
\end{tabular}

Nota: $N=106$; $B=$ coeficiente de regressão não padronizado; $S E B=$ erro padronizado; $\beta=$ coeficiente de regressão padronizado;

$R^{2}=$ coeficiente de determinação com variância explicada; $\Delta R^{2}=$ diferença na proporção da variância explicada. 
cada processo cognitivo no desempenho na FVF. Essas mudanças confirmaram-se nas análises de regressão múltipla (método stepwise), em que a contribuição dos escores para a produção total variou conforme o método de pontuação.

Para o método valor bruto, os três escores estratégicos contribuíram de forma independente para explicar a variabilidade no número de palavras produzidas, tendo sido controlados os efeitos das variáveis idade e escolaridade. O escore número de transições mostrou-se o melhor preditor do número de palavras evocadas (standardized $\beta=0,96$ ), seguido do número de agrupamentos e do tamanho destes. Já no método das taxas, apenas a taxa do tamanho médio dos agrupamentos colaborou significativamente para explicar a variabilidade no número de palavras produzidas, mesmo depois de controlada a contribuição da idade e da escolaridade das crianças. Apesar de significativa, é preciso considerar que a taxa do tamanho médio de agrupamento é a melhor preditora em um modelo que explica apenas $49 \%$ da variabilidade das palavras evocadas na tarefa. Além disso, o referido escore varia em direção oposta ao número total de palavras.

Adicionalmente, o método das taxas dos escores estratégicos é capaz de explicar apenas $49 \%$ da variabilidade na produção total de palavras, o que limita seu poder em avaliar o desempenho das crianças. Por sua vez, o método do valor bruto mostrou-se capaz de explicar $86 \%$ da variabilidade na produção total de palavras. Desse modo, o método de valor bruto apresentou maior capacidade de explicar a produção total de palavras pelas crianças, o que é condizente com a maior consistência psicométrica apresentada por esse método nas análises exploratórias de componente principal.

A análise exploratória de componente principal revelou que os escores estratégicos calculados pelo método valor bruto (Troyer et al., 1997) constituem um modelo explicativo psicometricamente consistente, que explica cerca de $90 \%$ da variabilidade observada. Além disso, para o método valor bruto, os fatores extraídos dão origem a dois componentes distintos, que podem ser identificados como organização (fator composto pelos escores número e tamanho de agrupamento) e flexibilidade (fator composto pelos escores de transição e agrupamento) (Koren et al., 2005). Essa organização fatorial está de acordo com o modelo teórico da busca estratégica, em que o desempenho na FVF envolve processos de organização (produção de palavras com base em subcategorias específicas de busca) e transição (mudança para uma nova categoria de busca quando a primeira foi esgotada) (Troyer et al., 1997).

Para o método das taxas, em contrapartida, a análise de componente principal apontou a existência de apenas um componente bipolar, que privilegia a organização à mudança do critério como estratégia de busca de palavras. Esse componente, que explica $74 \%$ da variabilidade observada, não é compatível com o modelo teórico de busca estratégica, que postula a participação tanto de processos de organização como de flexibilidade.

Em suma, a análise das propriedades psicométricas dos escores estratégicos revelou que o cálculo pelo método valor bruto (Troyer et al., 1997) apresentou mais evidências de validade baseadas na estrutura interna do instrumento se comparada à do método das taxas dos escores estratégicos (Sauzéon et al., 2004), além de melhor explicar a variabilidade do número de palavras evocadas (Gruenewald \& Lockhead, 1980). A avaliação da busca estratégica analisada pelo método de cálculo por taxa pode não mensurar de forma apropriada o desempenho de crianças, que envolve a participação de processos executivos de organização e flexibilidade (Troyer, 2000). A maior participação dos escores de transição e de agrupamento na explicação da variabilidade da produção total é condizente com a compreensão da FVF como medida que avalia predominantemente o funcionamento executivo. Tais resultados são corroborados por estudos anteriores, tanto em adultos (Shao et al., 2014; Troyer et al., 1997) como em crianças (Sauzéon et al., 2004; Takács et al., 2014), independentemente do método de cálculo utilizado.

Apesar da contribuição deste estudo, é preciso apontar suas limitações. Análises posteriores devem ser consideradas observando as mudanças nos escores e na produção por intervalo de tempo ao longo dos 60 segundos da tarefa, examinando os efeitos do desenvolvimento. Além disso, devem ser compreendidas as semelhanças e diferenças entre a fluência semântica e a fluência fonológica, sobretudo ao longo do desenvolvimento. Carecem, ainda, ser investigadas evidências de validade dos métodos de pontuação de escores estratégicos tendo em vista outras medidas clássicas em funções executivas e vocabulário, uma vez que o construto utilizado como referência é um modelo delineado com adultos.

\section{Referências}

Abwender, D. A., Swan, J. G., Bowerman, J. T., \& Connolly, S. W. (2001). Qualitative analysis of verbal fluency output: review and comparison of several scoring methods. Assessment, 8(3), 323-338. doi: 10.1177/107319110100800308

American Psychological Association (APA), American Educational Research Association (AERA), \& National Council on Measurement in Education (NCME) (1999). Standards for educational and psychological testing. Washington, D.C.: AERA.

Andrewe, D (2001). Neuropsychology: from theory to practice. Nova York: Psychology Press.

Banerjee, P., Grange, K., Steiner, D., \& White, A. (2011). Executive strategic processing during verbal fluency performance in children with phenylketonuria. Child Neuropsychology, 17(2), 105-117. doi: 10.1080/09297049.2010.525502

Begeer, S., Wierda, M., Scheeren, M., Teunisse, P., Koot, M., \& Geurts, M. (2014). Verbal fluency in children with autism spectrum disorders: clustering and switching strategies. Autism, 18(8), 1014-1018. doi: 10.1177/1362361313500381 
Charchat-Fichman, H., Oliveira, R. M., \& Morais, A. (2011). Performance of Brazilian children on phonemic and semantic verbal fluency tasks. Dementia Neuropsychology, 5(2), 78-84. doi: 10.1590/S1980-57642011DN05020004

Chertkow, H. \& Bub, D. (1990) Semantic memory loss in dementia of Alzheimer's type. Brain, 113(2), 397-417. doi: 10.1093/brain/113.2.397

Gonçalves, H. A. (2015). Clustering e switching na fluência verbal infantil: idade, tipo de escola e TDAH (Dissertação de Mestrado). Programa de Pós-Graduação em Psicologia, Pontifícia Universidade Católica do Rio Grande do Sul, Rio Grande do Sul, Brasil.

Gruenewald, P. J. \& Lockhead, G. R. (1980). The free recall of category examples. Journal of Experimental Psychology: Human Learning and Memory, 6(3), 225-241. doi: 10.1037/0278-7393.6.3.225

Kavé, G., Kigel, S., \& Kochva, R. (2008). Switching and clustering in verbal fluency tasks throughout childhood. Journal of Clinical and Experimental Neuropsychology, 30(3), 349-359. doi: 10.1080/13803390701416197

Koren, R., Kofman, O., \& Berger, A. (2005). Analysis of word clustering in verbal fluency of school-aged children. Archives of Clinical Neuropsychology, 20(8), 1087-1104. doi: 10.1016/j.acn.2005.06.012

Lezak, M. D., Howieson, D. B., Bigler, E. D., \& Tranel, D. (2012). Neuropsychological Assessment (5a Ed). Nova York: Oxford University Press.

Luria, A. R. (1973). The working brain. Nova York: Basic Books.

Martins, I. P., Vieira, R., Loureiro, C., \& Santos, M. E. (2007). Speech rate and fluency in children and adolescents. Child Neuropsychology, 13(4), 319-332. doi: 10.1080/09297040600837370

Mousinho, R. \& Correa, J. (2009). Conhecimento ortográfico na dislexia fonológica. In: T. Barbosa, C. C. Rodrigues, C. B. Melo, S. A. Capellini, \& L. M. Alves (Eds.). Temas em dislexia (pp. 33-45). São Paulo: Artes Médicas.

Nieto, A., Galtier, I., Barroso, J., \& Espinosa, G. (2008). Fluencia verbal en niños españoles en edad escolar: estúdio normativo piloto y análisis de las estrategias organizativas. Revista de Neurologia, 46(1), 2-6.

Oliveira, R. M., Mograbi, D. C., Gabrig, I. A., \& Charchat-Fichman, H. (2016). Normative data and evidence of validity for the Rey Auditory Verbal Learning Test, Verbal Fluency Test, and Stroop Test with Brazilian children. Psychology \& Neuroscience (Impresso), 9(1), 54-67. doi: 10.1037/pne0000041

Pawlowski, J., Trentini, C. M., \& Bandeira, D. R. (2007) Discutindo procedimentos psicométricos a partir da análise de um instrumento de avaliação neuropsicológica breve. Psico-USF, 12(2), 211-219. doi: 10.1590/S1413-82712007000200009

Raskin, S. H., Sliwinski, M. \& Borod, J. C. (1992). Clustering strategies on tasks of verbal fluency in Parkinson's disease. Neuropsychologia, 30(1), 95-99. doi: 10.1016/0028-3932(92)90018-H

Riva, D., Nichelli, F., \& Devoti, M. (2000). Developmental aspects of verbal fluency and confrontation naming in children. Brain and Language, 71(2), 267-284. doi: 10.1006/brln.1999.2166

Ruff, R. M., Light, R. H., \& Parker, S. B. (1997) The psychological construct of word fluency. Brain and Language, 57(3), $394-405$. doi: 10.1006/brln.1997.1755

Sauzéon, H., Lestage, P., Raboutet, C., N’Kaoua, B., \& Claverie, B. (2004). Verbal fluency output in children aged 7-16 as a function of the production criterion: qualitative analysis of clustering, switching processes, and semantic network exploitation. Brain and Language, 89(1), 192-202. doi: 10.1016/S0093-934X(03)00367-5

Shao, Z., Janse, E., Visser, K., \& Meyer, A. S. (2014). What do verbal fluency tasks measure? Predictors of verbal fluency performance in older adults. Frontiers in Psychology, 5, 772. doi:10.3389/fpsyg.2014.00772

Strauss, E., Shermann, E. M., \& Spreen, O. (2006) A compendium of neuropsychological tests. Administration Norm and Comentary. (3a ed.). Nova York: Oxford University Press.

Snyder, H.R. \& Munakata, Y. (2010). Becoming self-directed: abstract representations support endogenous flexibility in children. Cognition, 116(2), 155-167. doi: 10.1016/j.cognition.2010.04.007

Tallberg, I. M., Carlsson, S., \& Lieberman, M. (2011). Children's word fluency strategies. Scandinavian Journal of Psychology, 52(1), 35-42. doi: 10.1111/j.1467-9450.2010.00842.x

Takács, A., Kóbor, A., Tárnok, Z., \& Csépe, V. (2014). Verbal fluency in children with ADHD: strategy using and temporal properties. Child Neuropsychology, 20(4), 415-429. doi: 10.1080/09297049.2013.799645

Thompson, B. \& Daniel, L.G. (1996). Factor analytic evidence for the construct validity of scores: a historical overview and some guidelines. Educational and Psychological Measurement, 56(2), 197-208. doi: 10.1177/0013164496056002001

Troyer, A. K. (2000). Normative data for clustering and switching on verbal fluency tasks. Journal of Clinical and Experimental Neuropsychology, 22(3), 370-378. doi: 10.1076/1380-3395(200006)22:3;1-v;ft370

Troyer, A. K., Moscovitch, M., \& Winocur, G. (1997). Clustering and switching as two components of verbal fluency: evidence from younger and older healthy adults. Neuropsychology, 11(1), 138-146. doi: 10.1037/0894-4105.11.1.138

Troyer, A. K., Moscovitch, M., Winocur, G., Alexander, M. P., \& Stuss, D. (1998). Clustering and switching on verbal fluency: the effects of focal frontal-temporal-lobe lesions. Neuropsychologia, 36(6), 499-504. doi: 10.1016/S0028-3932(97)00152-8

Viaro, M. E. \& Guimarães-Filho, Z. O. (2007). Análise quantitativa da frequência dos fonemas e estruturas silábicas portuguesas. Estudos Lingïísticos, XXXVI(1), 27-36.

\section{Sobre as autoras}

Priscila do Nascimento Marques é Doutoranda em Psicologia Cognitiva pelo Programa de Pós-Graduação em Psicologia da Universidade Federal do Rio de Janeiro (PPGP/UFRJ) e Professora substituta do Departamento de Psicometria do Instituto de Psicologia da UFRJ.

Jane Correa é Doutora em Psicologia do Desenvolvimento (University of Oxford) e Professora titular do Departamento de Psicologia Geral e Experimental e da Pós-Graduação em Psicologia do Instituto de Psicologia da UFRJ.

Rosinda Martins Oliveira é Doutora em Psicologia Experimental (University of Oxford) e Professora adjunta do Departamento de Psicometria do Instituto de Psicologia da UFRJ.

Helenice Charchat-Fichman é Doutora em Neurociências e Comportamento pela Universidade de São Paulo (USP) e Professora adjunta do Departamento de Psicologia da Pontifícia Universidade Católica do Rio de Janeiro (PUC-Rio) (Graduação e Pós-Graduação). 